\title{
Finite-Difference Solution of the Helmholtz Equation Based on Two Domain Decomposition Algorithms
}

\author{
Wensheng Zhang ${ }^{1}$, Yunyin Dai ${ }^{2}$ \\ ${ }^{1}$ Institute of Computational Mathematics and Scientific/Engineering Computing, LSEC, Beijing, China \\ ${ }^{2}$ Academy of Mathematics and Systems Science, Chinese Academy of Sciences, Beijing, China \\ Email: zws@lsec.cc.ac.cn, daiyy@lsec.cc.ac.cn
}

Received July 2013

\begin{abstract}
In this paper, wave simulation with the finite difference method for the Helmholtz equation based on the domain decomposition method is investigated. The method solves the problem by iteratively solving subproblems defined on smaller subdomains. Two domain decomposition algorithms both for nonoverlapping and overlapping methods are described. More numerical computations including the benchmark Marmousi model show the effectiveness of the proposed algorithms. This method can be expected to be used in the full-waveform inversion in the future.
\end{abstract}

Keywords: Finite Difference; Domain Decomposition; Nonoverlapping; Overlapping; Helmholtz Equation; Preconditioner

\section{Introduction}

The numerical solution of acoustic wave equation is an important problem. The Helmholtz equation is the version of acoustic wave equation in the frequency domain. It has applications in seismic wave propagation, imaging and inversion. In the geophysical frequency-domain inversion, one needs to do forward modeling which means solving the Helmholtz equation. During the inversion process, the synthetic model is continuously updated until a convergence is reached. Thus numerical methods for solving the Helmholtz equation have been under active research during the past few decades. The finite element method and the finite difference method have been used successfully for this problem. The discretization of the 2D Helmholtz for mid-frequency and highfrequency problems may lead to a large linear system because of the requirement of ten points per wavelength. This makes the problem even harder to solve. Direct methods easily suffer from inacceptable computational work. So many iterative methods for the Helmholtz equation have been developed, for instance, see [1-6]. As the resulting system is non-Hermitian and indefinite, a good preconditioner is necessary for the iterative methods. Various preconditioners have been proposed [5-11], for example, a tensor product preconditioner [6], the incomplete factorization preconditioner [7] and the Laplacian preconditioner [8,9]. We will use the shifted-Laplacian preconditioner in this paper [9].

The domain decomposition method (DDM) is an effective technique for solving large-scale problems [12-
22]. It splits the whole computational domain into several smaller subdomains and solves a sequence of similar subproblems on these subdomains. The number and size of subdomains can now be chosen so as to enable direct methods to solve the subproblems. Between adjacent subdomains the boundary conditions are adjusted iteratively by transmission conditions. For the boundary of whole computational domain, absorbing boundary conditions (ABCs) are required. There exist several ABC methods, for instance, the paraxial approximation method [23] and the perfectly matched layer method [24]. In this paper, we use the former as our computational domain is a rectangular domain.

In this paper, we focus on solving the Helmholtz with the finite difference method based on the nonoverlapping and overlapping DDM algorithms. More numerical computations demonstrate the correctness of the algorithms presented in this paper. The method will be used in the frequency-domain inversion in the future.

\section{Theory}

\subsection{Finite Difference Scheme}

The 2-D acoustic wave equation can be written as

$$
\frac{1}{v(x, z)^{2}} \frac{\partial^{2} u}{\partial t^{2}}-\frac{\partial^{2} u}{\partial x^{2}}-\frac{\partial^{2} u}{\partial z^{2}}=g(x, z),
$$

with the absorbing boundary conditions

$$
\frac{\partial u}{\partial n}+\frac{1}{v(x, z)} \frac{\partial u}{\partial t}=0
$$


where $v(x, z)$ is the velocity of media and $u(x, z, t)$ is the wavefield, $g(x, z)$ is the source term. In the frequency domain (1) can be written as

$$
-\frac{\partial^{2} u}{\partial x^{2}}-\frac{\partial^{2} u}{\partial z^{2}}-k^{2}(x, z) u=g(x, z),
$$

where $k=\omega / v(x, z)$ is the wave number and $\omega=2 \pi f$ is the angular frequency. The boundary condition in the frequency domain is

$$
\frac{\partial u}{\partial n}-i k(x, y) u=0,
$$

where $n$ is the outward normal of the boundary and $i=\sqrt{-1}$ is the imagine unit. We use the second-order difference scheme to discrete (3) and (4) and the result can be written as a linear system

$$
A u=b, \quad A \in C^{N \times N}, u, b \in C^{N},
$$

where $N=N_{x} N_{y}$ is the total number of wavefield $u$ on the computational domain $\Omega_{h}$, and $N_{x}$ and $N_{y}$ are the discretization number along $x$ and $z$ directions respectively. The matrix $A$ is a complex matrix as the boundary condition contains complex number. Moreover, it is non-positive and non-Hermitian matrix. Usually we use the Krylov iterative methods to solve (5) as $A$ is a large-scale sparse matrix. The Bi-CGSTAB algorithm $[25,26]$ is a good choice. The following is the Bi-CGSTAB algorithm for solving $A x=b$.

Algorithm 1 [Bi-CGSTAB algorithm]

Step 1. Give the matrix $A$, vector $b$ and initial value $x_{0}$, the maximal iterative number $k_{\max }$ and the tolerance error $\varepsilon_{\text {tol }}$, the preconditioned matrix $M$, compute $r_{0}=b-A x_{0}$ and set $k=1$ and $\tilde{r}_{0}=r_{0}$;

Step 2. If $k \leq k_{\max }$ and $\varepsilon>\varepsilon_{\text {tol }}$, turn to step 3; otherwise stop and output $x_{k}$;

Step 3. Compute $\rho_{k-1}=r_{k-1}^{T} r_{k-1}$, if $\rho_{k-1}=0$ or $\omega_{k-1}=0$ then stop; otherwise turn to step 4;

Step 4. If $k=1$ then $p_{1}=r_{0}$, otherwise compute

$$
\beta_{k-1}=\frac{\rho_{k-1} \alpha_{k-1}}{\rho_{k-2} \omega_{k-1}}, \quad p_{k}=r_{k-1}+\beta_{k-1}\left(p_{k-1}-\omega_{k-1} V_{k-1}\right) ;
$$

Step 5. Solve the system $M \hat{p}=p$ and compute

$$
V_{k}=A \hat{p}, \quad \alpha_{k-1}=\frac{\rho_{k-1}}{r^{T} V_{k}}, \quad s_{k}=r_{k-1}-\alpha_{k} V_{k} ;
$$

Step 6. Set $\varepsilon=\left\|s_{k}\right\|$, if $\varepsilon>\varepsilon_{\text {tol }}$ then $x_{k}=x_{k-1}+\alpha_{k} \hat{p}$, otherwise stop and output $x_{k}$;

Step 7. Solve the systems $M \hat{s}=s$ and $t=A \hat{s}$, then compute

$$
\omega_{k}=\frac{t^{T} s \rho_{k-1}}{t^{T} t}, x_{k}=x_{k-1}+\alpha_{k} p_{k}+\omega_{k} \hat{s},
$$

and set $\varepsilon=\left\|r_{k}\right\|$, let $k=k+1$, turn to step 2 .

For the preconditioner matrix $M$, we adopt the shifted-
Laplace preconditioned method [9]

$$
M_{S L}=-\frac{\partial^{2}}{\partial x^{2}}-\frac{\partial^{2}}{\partial y^{2}}+\alpha k^{2}(x, y),
$$

where $\operatorname{Re}(\alpha)>0$ and $\operatorname{Im}(\alpha)>0$. A typical choice for $\alpha$ is $\alpha=i$, named complex shifted-Laplace preconditioner.

\subsection{Two Domain Decomposition Algorithms}

In this subsection we discuss how to solve the problem (3) and (4) with DDM, including the nonoverlapping algorithm and overlapping algorithm. First of all, we consider the nonoverlapping problem. We divide the computational domain $\Omega$ into $N$ non-overlapped subdomains $\Omega_{m}, \quad m=1, \cdots, N$. Denote $u^{n, m}$ be the value of $u$ at $n$ th iteration and on the $m$ th subdomain $\Omega_{m}$. Obviously the division satisfies

$$
\bar{\Omega}=\bigcup \bar{\Omega}_{i}, \quad \Omega \Omega_{i} \Omega_{j}=\phi, \quad \Gamma_{i j}=\partial \Omega_{i} \bigcap \partial \Omega_{j}, \quad(i \neq j) .
$$

Given the iteration value $u^{0, m}, m=1, \cdots, N$, solve the following system iteratively:

$$
\begin{gathered}
-\frac{\partial^{2} u^{n, m}}{\partial x^{2}}-\frac{\partial^{2} u^{n, m}}{\partial z^{2}}-k^{2} u^{n, m}=g\left(x_{i j}, y_{i j}\right),(x, y) \in \Omega_{m} \\
\frac{\partial u^{n, m}}{\partial n_{m}}-i k u^{n, m}=0, \quad(x, y) \in \partial \Omega \bigcap \partial \Omega_{m}, \\
\frac{\partial u^{n, m}}{\partial n_{m}}-i k u^{n, m}=-\frac{\partial u^{n-1, r}}{\partial n_{r}}-i k u^{n-1, r}, \\
(x, y) \in \Gamma_{m r}=\partial \Omega_{m} \bigcap \partial \Omega_{r} .
\end{gathered}
$$

The last Equation (12) is the interface equation. Using the standard five-point difference scheme, we obtain the following system

$$
A^{n, m} u^{n, m}=b^{n, n}, \quad m=1, \cdots, N .
$$

In the iterations, the $u^{n-1, m}$ is assumed to be known and is used in the interface equation. Thus we can give the following nonoverlapping DDM algorithm.

Algorithm 2 [Nonoverlapping DDM algorithm]

Step 1. Select initial value $u^{0, m}$ and set $n=0$.

Step 2. Obtain $b^{n, m}$ by solving the interface equation discretized from (12).

Step 3. Solve the system (14).

Step 4. Set $n=n+1$, turn to step 2 .

In the following we consider the overlapping DDM. We still divide the computational domain $\Omega$ into $N$ subdomains $\Omega_{i}, \quad i=1, \cdots, N$ :

$$
\bar{\Omega}=\bigcup \bar{\Omega}_{i},
$$

but now any two adjacent subdomains are overlapped, i.e.,

$$
\Omega_{m} \bigcap \Omega_{r} \neq \phi
$$


For simplicity we consider the case of two subdomains, i.e., $N=2$ and $\Omega_{1} \bigcap \Omega_{2} \neq \phi$. Notice that the boundary

$\Gamma_{i}=\partial \Omega_{i} \backslash\left(\partial \Omega \bigcap \partial \Omega_{i}\right)$ (17) doesn't belong to the subdomain $\Omega_{i}$. When the iteration for the problem convergences, the following conditions are true obviously

$$
\begin{gathered}
u^{n, m}-u^{n-1, m} \rightarrow 0, \\
\frac{\partial u^{n, m}}{\partial n_{i}}-\frac{\partial u^{n-1, m}}{\partial n_{i}} \rightarrow 0, \quad n \rightarrow+\infty,
\end{gathered}
$$

To keep the symmetry of the matrix on the subdomains, we construct the auxiliary equation

$$
\frac{\partial u^{n, m}}{\partial n_{m}}-i k u^{n, m}=\frac{\partial u^{n-1, r}}{\partial n_{r}}-i k u^{n-1, r}
$$

Thus we get the similar system

$$
\tilde{A}^{n, m} u^{n, m}=\tilde{b}^{n, n}, \quad m=1, \cdots, N .
$$

Now we can give the following overlapping DDM algorithm.

Algorithm 3 [Overlapping DDM algorithm]

Step 1. Set $n=0$ and select initial value $u^{n, m}$;

Step 2. Solve the auxiliary Equation (21);

Step 3. Extrapolate $u^{n, m}$ according to the following formulation:

$$
\begin{gathered}
\left.u^{n, m}\right|_{\Omega_{m}}=u^{n, m},\left.u^{n, m}\right|_{\Omega \backslash \Omega_{m}}=u^{n, m}, \\
u^{n}=\frac{1}{N} \sum_{i=1}^{N} u^{n, m} ;
\end{gathered}
$$

Step 4. Set $n=n+1$ and turn to step 2 .

\section{Numerical Computations}

For testing the correctness of the discretized finite difference schemes, we solve the problem without using DDM first. The first model is a homogeneous model with constant wave number. The computational domain is a square $\Omega=(0,1)^{2}$, and the source $g(x, y)$ is defined as the following $\delta$ function:

$$
\delta(x, y)= \begin{cases}1, & x=1 / 2, y=1,2 . \\ 0, & \text { other. }\end{cases}
$$

We use the preconditioned Bi-CGSTAB method to solve the problem. Figure 1, Figures $\mathbf{2}$ and $\mathbf{3}$ are the simulation results for wave number 20,30 and 40 respectively. We can see that the obtained waveform in these figures is very clear. We also can see that the wave has more vibration as $k$ increases. Next we consider a three-layered model shown in Figure 4. The velocity from top to bottom is $2700 \mathrm{~m} / \mathrm{s}, 1500 \mathrm{~m} / \mathrm{s}$ and $2100 \mathrm{~m} / \mathrm{s}$ respectively. The computational domain is a rectangle domain: $\Omega=(0,600) \times(0,1000)$, and $g(x, y)$ is the following $\delta$ function

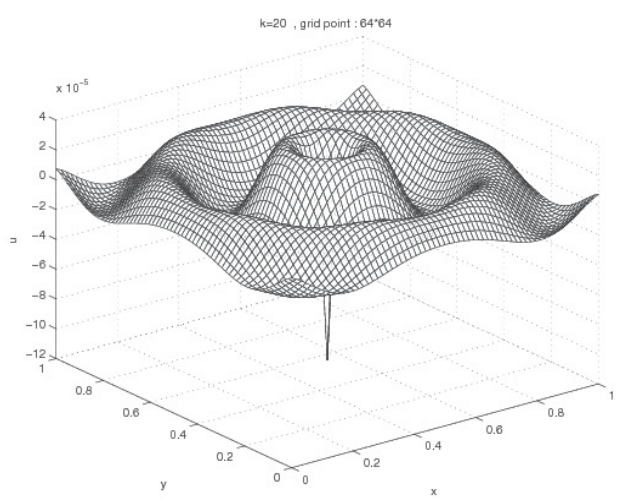

Figure 1. Wavefield for $k=20$ obtained by the preconditioned Bi-CGSTAB method. The DDM is not used.

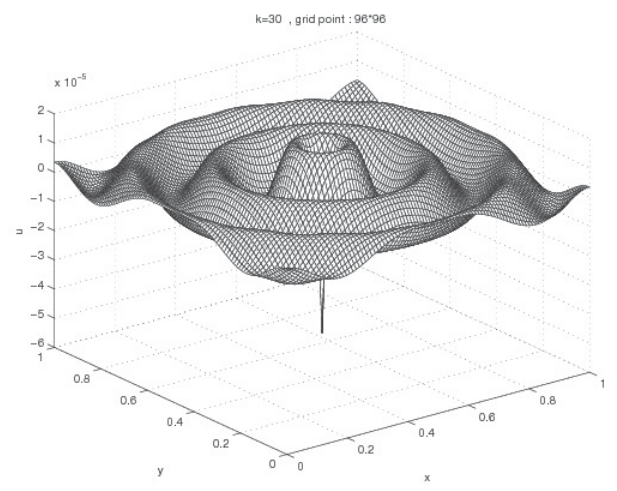

Figure 2. Wavefield for $k=30$ obtained by the preconditioned Bi-CGSTAB method. The DDM is not used.

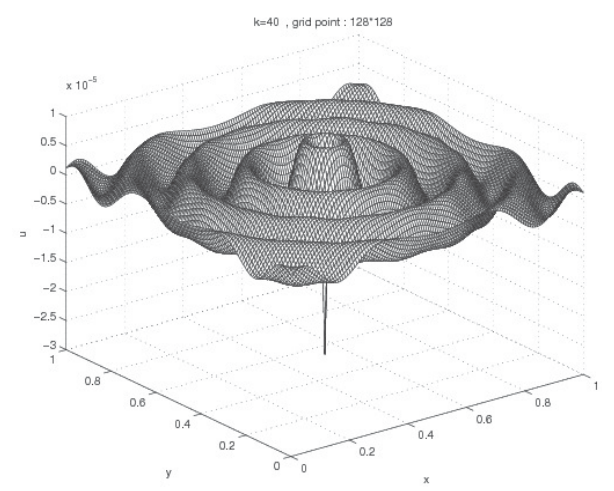

Figure 3. Wavefield for $k=40$ obtained by the preconditioned Bi-CGSTAB method. The DDM is not used.

$$
\delta(x, y)= \begin{cases}1, & x=300, y=1,2 . \\ 0, & \text { other. }\end{cases}
$$

Figures 5 and $\mathbf{6}$ are the simulation results for this model with wave number 20 and 30 respectively. Our analysis shows the results are right.

Now we solve the problem with the nonoverlapping DDM. We consider a square domain $\Omega=(0,1)^{2}$. We divide this domain into two subdomains. Figure $\mathbf{7}$ is the 


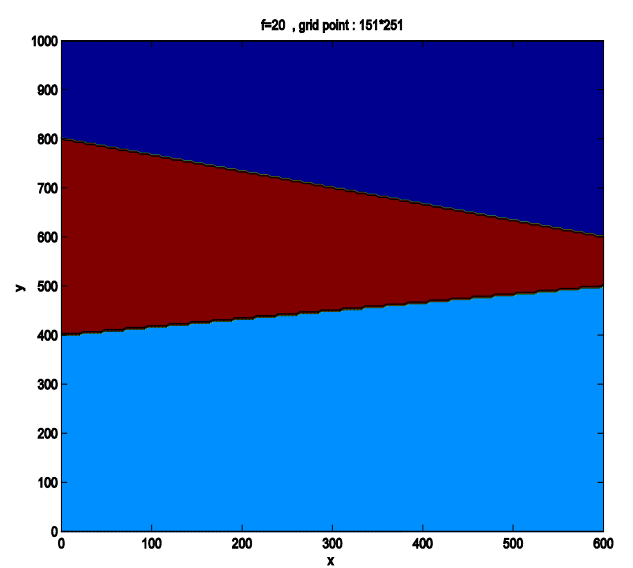

Figure 4. A three-layered model with velocity $2700 \mathrm{~m} / \mathrm{s}$, $1500 \mathrm{~m} / \mathrm{s}$ and $2100 \mathrm{~m} / \mathrm{s}$ from top to bottom.

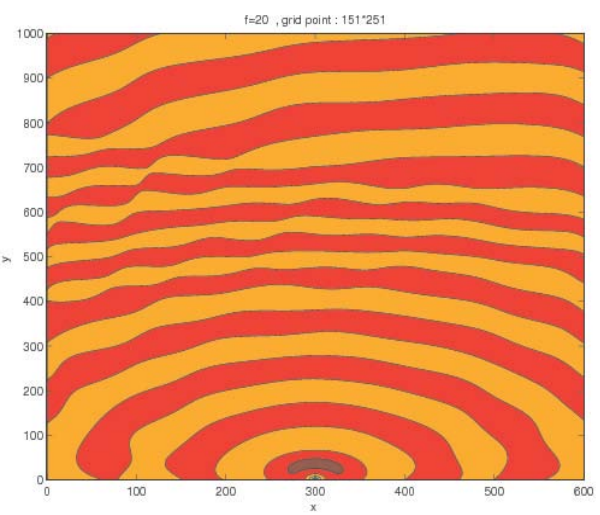

Figure 5. Wavefield contour for $f=20 \mathrm{~Hz}$ obtained by the preconditioned Bi-CGSTAB method. The DDM is not used.

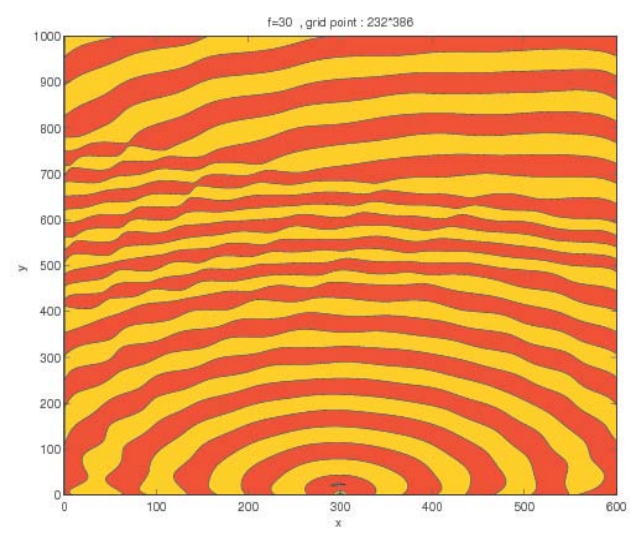

Figure 6. Wavefield contour $f=30 \mathrm{~Hz}$ obtained by the preconditioned Bi-CGSTAB method. The DDM is not used.

wavefield result for $f=4 \mathrm{~Hz}$ obtained by the nonoverlapping DDM algorithm with two subdomains. For a L-type model we divide it into three subdomains. Figure 8 is the corresponding simulation result for $f=4 \mathrm{~Hz}$ obtained by the nonoverlapping DDM algorithm with three subdo- mains. Figure 9 is result for $f=4 \mathrm{~Hz}$ obtained by the nonoverlapping DDM method with four equal square subdomains for a square domain.

Next we solve the problem with the overlapping DDM algorithm. The velocity media is $2100 \mathrm{~m} / \mathrm{s}$. The location of the pulse is at $(x, y)=(3,2.5)$. The frequency is

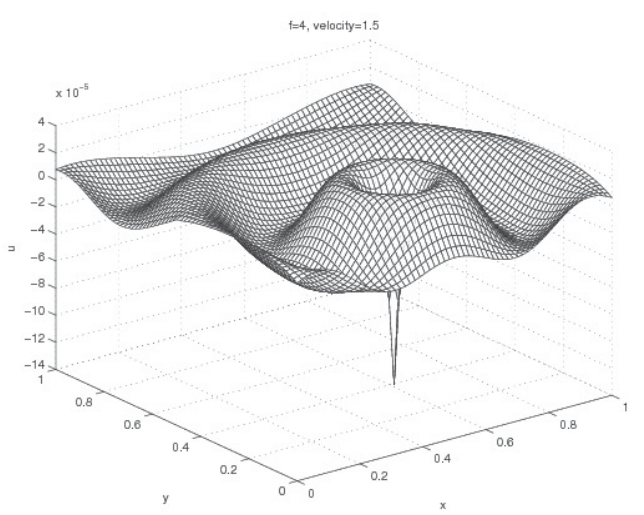

Figure 7. Wavefield for $f=4 \mathrm{~Hz}$ obtained by the nonoverlapping DDM algorithm. The square computational domain is divided into two subdomains up and down.

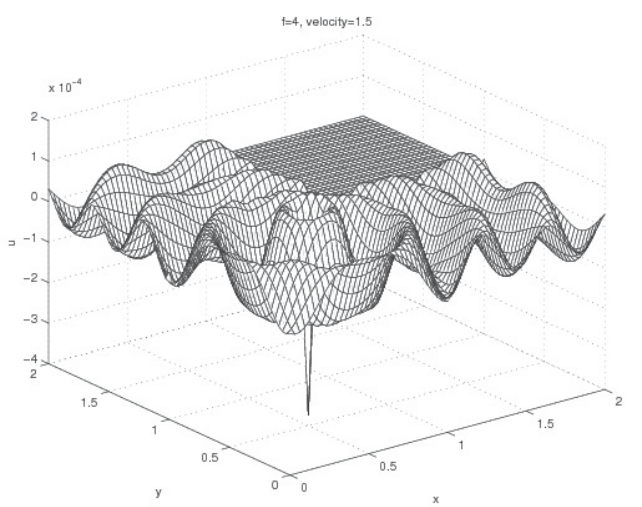

Figure 8. Wavefield for $f=4 \mathrm{~Hz}$ obtained by the nonoverlapping DDM algorithm. The L-type computational domain is divided into three square subdomains.

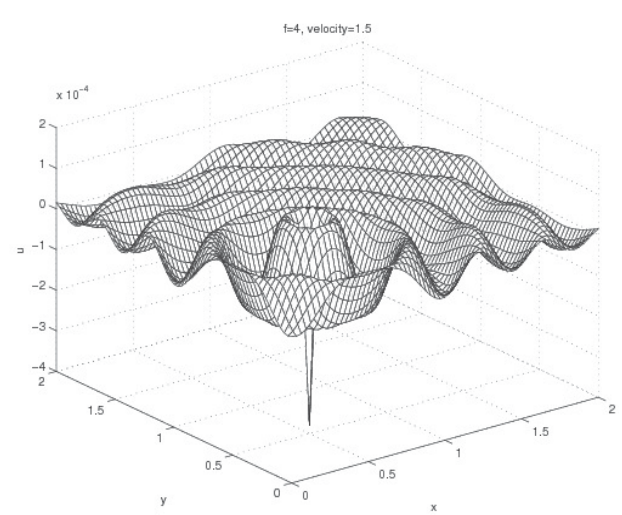

Figure 9. Wavefield for $f=4 \mathrm{~Hz}$ obtained by the nonoverlapping DDM algorithm. The square computational domain is divided into four equal square subdomains. 
$f=1 \mathrm{~Hz}$. Figure 10 is the wavefield obtained by the overlapping DDM algorithm with two equal square subdomains up and down. Figure 11 is the wavefield obtained by the overlapping DDM algorithm with three square subdomains for an L-type domain. Figure $\mathbf{1 2}$ is the

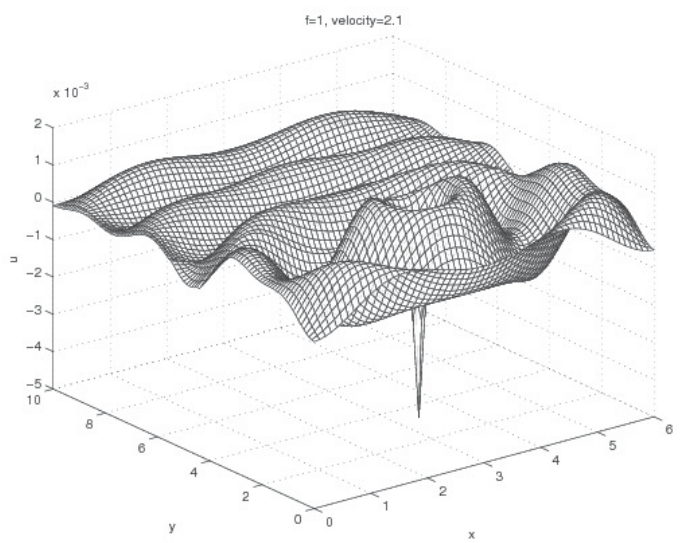

Figure 10. Wavefield for $f=1 \mathrm{~Hz}$ obtained by the overlapping DDM algorithm. The computational domain is divided into two equal subdomains up and down.

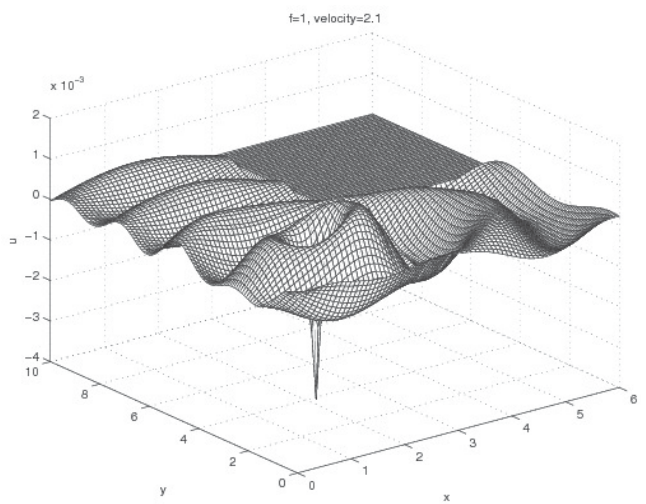

Figure 11. Wavefield for $f=1 \mathrm{~Hz}$ obtained by the overlapping DDM algorithm. The square computational domain is divided into three equal square subdomains.

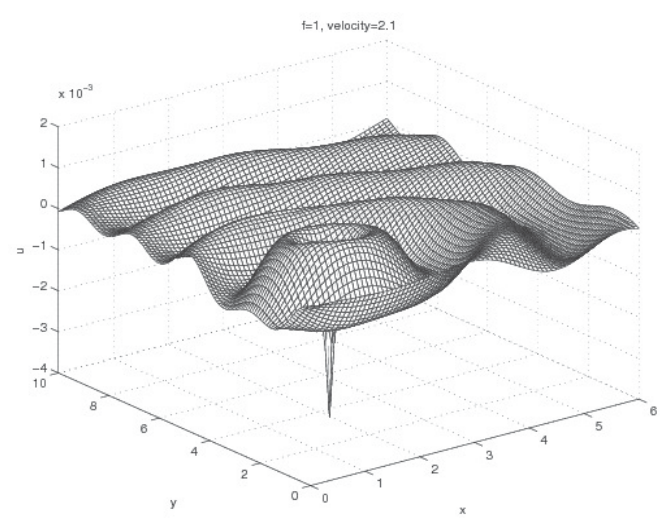

Figure 12. Wavefield for $f=1 \mathrm{~Hz}$ obtained by the overlapping DDM algorithm. The square computational domain is divided into four equal subdomains. wavefield obtained by the overlapping DDM algorithm with four equal square subdomains for a square domain.

Finally we consider a typical inhomogeneous model named Marmousi model which is usually used to test the ability of seismic migration and inversion [27]. The velocity is shown in Figure 13. The velocity varies from $1500 \mathrm{~m} / \mathrm{s}$ to $5500 \mathrm{~m} / \mathrm{s}$. We select a part of this model to simulate wave propagation. Figure $\mathbf{1 4}$ is the wavefield contour obtained by the preconditioned Bi-CGSTAB method for $f=5 \mathrm{~Hz}$ and the DDM algorithm is not used. Figure 15 is the wavefield contour obtained by the nonoverlapping DDM algorithm with two subdomains. Figure 16 is the wavefield contour obtained by the overlapping DDM algorithm with two subdomains. Figure 17 is the similar result but for $f=20 \mathrm{~Hz}$. Comparing Figures 15 and 16 with Figure 14, we know that they almost the same which are just we expect.

\section{Conclusion}

The acoustic wave equation in the frequency domain is solved by the finite difference method based on the domain decomposition method. The discritizaiton of the

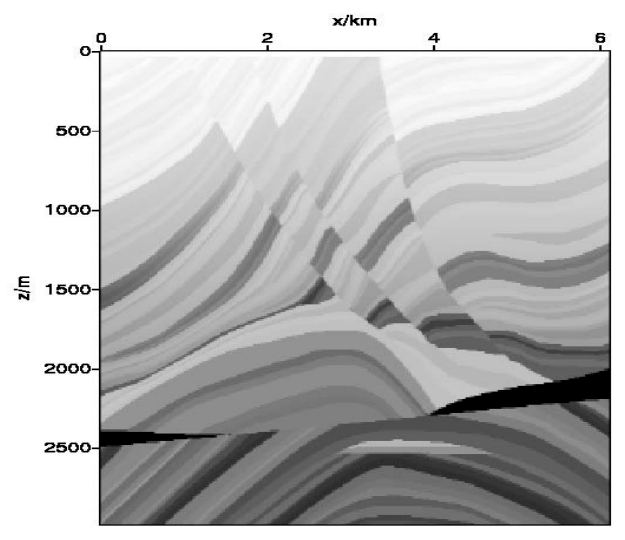

Figure 13. Marmousi model.

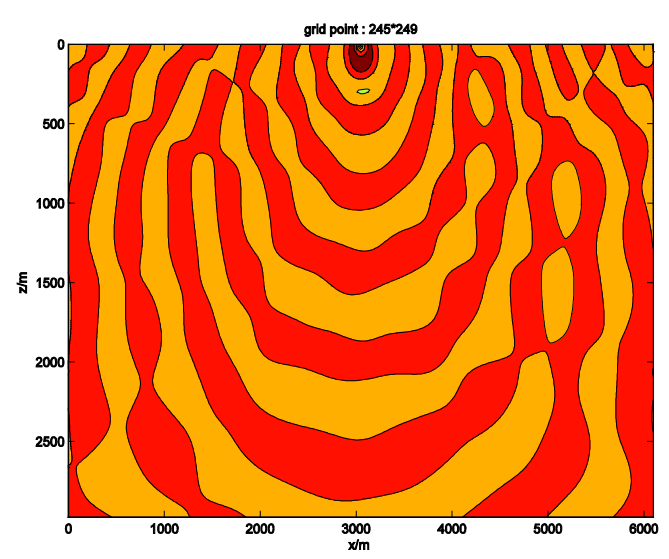

Figure 14. Wavefield contour for $f=5 \mathrm{~Hz}$ obtained by the preconditioned Bi-CGSTAB algorithm. The DDM algorithm is not used. 


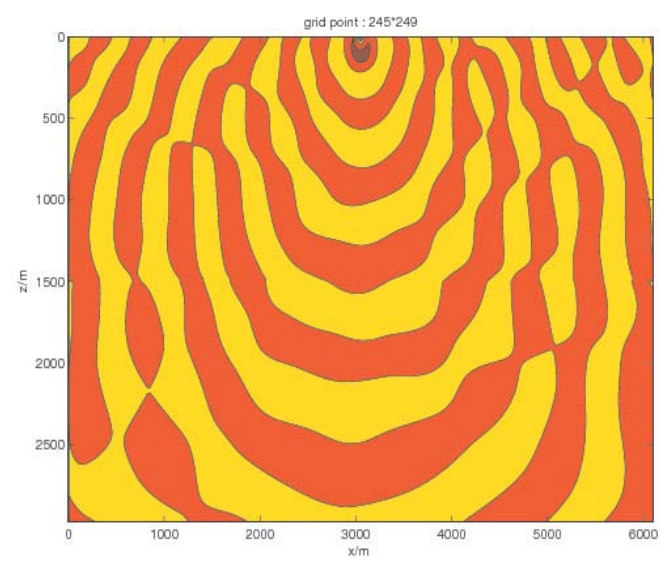

Figure 15. Wavefield contour for $f=5 \mathrm{~Hz}$ by Bi-CGSTAB preconditioned method. The nonoverlapping DDM algorithm with two subdomains is used.

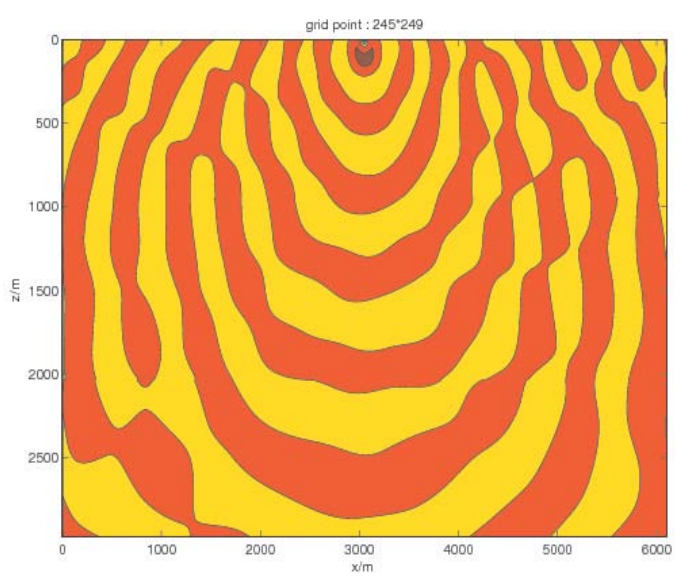

Figure 16. Wavefield contour for $f=5 \mathrm{~Hz}$ obtained by Bi-CGSTAB preconditioned method. The overlapping DDM algorithm with two subdomains is used.

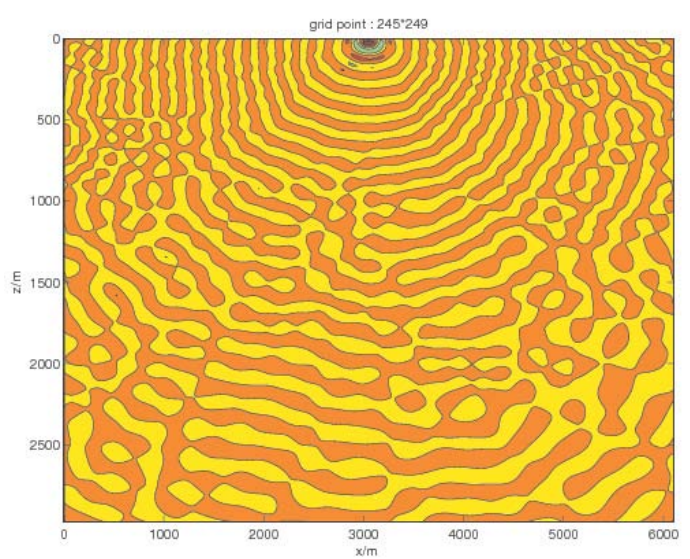

Figure 17. Wavefield contour for $f=20 \mathrm{~Hz}$ obtained by Bi-CGSTAB preconditioned method. The overlapping DDM algorithm with two subdomains is used.

problem leads to a sparse system which is solved by the complex shifted-Laplace preconditioned Bi-CGSTAB iteration method. Two DDM algorithms both for nonoverlapping and overlapping method are given. Many numerical computational examples including the complex Marmousi model are implemented which show the correctness and effectiveness of the algorithms presented in this paper. This method can be used in the full-waveform inversion. It can sometimes reduce the computational complexity.

\section{Acknowledgements}

This research is supported by the State Key project with grant number 2010CB731505 and the Foundation of National Center for Mathematics and Interdisciplinary Sciences, Chinese Academy of Sciences. The computations are implemented in the State Key Laboratory of Scientific and Engineering Computing (LSEC).

\section{REFERENCES}

[1] A. Bayliss, C. I. Goldstein and E. Turkel, "The Numerical Solution of the Helmholtz Equation for Wave Propagation Problems in Underwater Acoustics," Computers and Mathematics with Applications, Vol. 11, No. 7-8, 1985, pp. 655-665.

http://dx.doi.org/10.1016/0898-1221(85)90162-2

[2] R. E. Plexxis, "A Helmholtz Iterative Solver for 3D Seismic-Imaging Problems,” Geophysics, Vol. 72, No. 5, 2007, pp. SM185-SM197.

http://dx.doi.org/10.1190/1.2738849

[3] A. Bayliss, C. I. Goldstein and E. Turkel, “An Iterative Method for the Helmholtz Equation,” Journal of Computational Physics, Vol. 49, No. 3, 1983, pp.443-457. http://dx.doi.org/10.1016/0021-9991(83)90139-0

[4] C. W. Oosterlee and T. Washio, "Iterative Solution of the Helmholtz Equation by a Second Order Method," SIAM Journal on Matrix Analysis and Applications, Vol. 21, No. 1, 1999, pp. 209-229. http://dx.doi.org/10.1137/S0895479897316588

[5] Y. A. Erlangga, “Advances in Iterative Methods and Preconditioners for the Helmholtz Equation," Archives of Computational Methods in Engineering, Vol. 15, No. 1, 2008, pp. 37-66. http://dx.doi.org/10.1007/s11831-007-9013-7

[6] R.-E. Plessix and W. A. Mulder, "Separation of Variables as a Preconditioner for an Iterative Helmholtz Solver," Applied Numerical Mathematics, Vol. 44, No. 3, 2003, pp. 385-400.

http://dx.doi.org/10.1016/S0168-9274(02)00165-4

[7] M. M. M. Made, "Incomplete Factorization-Based Preconditionings for Solving the Helmholtz Equation,” International Journal for Numerical Methods in Engineering, Vol. 50, No. 5, 2001, pp. 1077-1101. http://dx.doi.org/10.1002/1097-0207(20010220)50:5<107 7::AID-NME65>3.0.CO;2-P

[8] N. Umetani, S. P. Maclachlan and C. W. Oosterlee, “A Multigrid-Based Shifted Laplacian Preconditioner for a Fourth-Order Helmholtz Discretization,” Numerical Li- 
near Algebra with Applications, Vol. 16, No. 8, 2009, pp. 603-626. http://dx.doi.org/10.1002/nla.634

[9] T. Airaksinen, E. Heikkola, A. Pennanen and J. Toivanen, "An Algebraic Multigrid Based Shifted-Laplacian Preconditioner for the Helmholtz Equation," Journal of Computational Physics, Vol. 226, No. 1, 2007, pp. 11961210. http://dx.doi.org/10.1016/j.jcp.2007.05.013

[10] Y. A. Erlangga, C. Vuik and C. W. Oosterlee, “On a Class of Preconditioners for Solving the Helmholtz Equation,” Applied Numerical Mathematics, Vol. 50, No. 3-4, 2004, pp. 409-425. http://dx.doi.org/10.1016/j.apnum.2004.01.009

[11] Y. A. Erlangga, C. W. Oosterlee and C. Vuik, “A Novel Multigrid Based Preconditioner for Heterogeneous Helmholtz Problems," SIAM Journal on Scientific Computing, Vol. 27, No. 4, 2006, pp. 1471-1492. http://dx.doi.org/10.1137/040615195

[12] T. F. Chan and T. P. Mathew, "Domain Decomposition Algorithms,” Acta Numerica, Vol. 3, 1994, pp. 61-143. http://dx.doi.org/10.1017/S0962492900002427

[13] J. D. Benamou and B. Despres, “A Domain Decomposition Method for the Helmholtz Equation and Related Optimal Control Problems," Journal of Computational Physics, Vol. 136, No. 1, 1997, pp. 62-68. http://dx.doi.org/10.1006/jcph.1997.5742

[14] B. Smith, P. Bjorstad and W. Grop, "Domain Decomposition: Parallel Multilevel Methods for Elliptic Partial Differential Equations," Cambridge University Press, Cambridge, 1996.

[15] A. Quarteroni and A. Valli, "Domain Decomposition Methods for Partial Differential Equations,” Oxford Science Publications, Oxford, 1999.

[16] A. Tosseli and O. Widlund, "Domain Decomposition Methods-Algorithms and Theory," Springer, Berlin, 2005.

[17] J. Xu, "Iterative Methods by Space Decomposition and Subspace Correction,” SIAM Review, Vol. 34, No. 4, 1992, pp. 581-613. http://dx.doi.org/10.1137/1034116

[18] J. Xu and J. Zou, "Some Nonoverlapping Domain Decomposition Methods," SIAM Review, Vol. 40, No. 4,
1998, pp. 857-914. http://dx.doi.org/10.1137/S0036144596306800

[19] S. Kim, "Domain Decomposition Iterative Procedures for Solving Scalar Waves in the Frequency Domain,” Numerische Mathematik, Vol. 79, No. 2, 1998, pp. 231-259. http://dx.doi.org/10.1007/s002110050339

[20] S. Larsson, "A Domain Decomposition Method for the Helmholtz Equation in a Multilayer Domain," SIAM Journal on Scientific Computing, Vol. 20, No. 5, 1999, pp. 1713-1731. http://dx.doi.org/10.1137/S1064827597325323

[21] F. Magoulès, F. X. Roux and S. Salmon, "Optimal Discrete Transmission Conditions for a Nonoverlapping Domain Decomposition Method for the Helmholtz Equation,” SIAM Journal on Scientific Computing, Vol. 25, No. 5, 2004, pp. 1497-1515. http://dx.doi.org/10.1137/S1064827502415351

[22] E. Heikkola, T. Rossi and J. Toivaned, “A Parallel Fictitious Domain Method for the Three-Dimensional Helmholtz Equation,” SIAM Journal on Scientific Computing, Vol. 24, No. 5, 2003, pp. 1567-1588. http://dx.doi.org/10.1137/S1064827500370305

[23] R. Clayton and B. Engquist, "Absorbing Boundary Conditions for Acoustic and Elastic Wave Equations,” Bulletin of the Seismological Society of America, Vol. 67, No. 6, 1977, pp. 1529-1540.

[24] J. P. Berenger, "A Perfectly Matched Layer for Absorbing of Electromagnetic Waves,” Journal of Computational Physics, Vol. 114, No. 2, 1994, pp. 185-200. http://dx.doi.org/10.1006/jcph.1994.1159

[25] H. A. van der Vorst, "Bi-CGSTAB: A Fast and Smoothly Converging Variant of Bi-CG for the Solution of Nonsymmetric Linear Systems", SIAM Journal on Scientific and Statistical Computing, Vol. 13, No. 2, 1992, pp. 631644. http://dx.doi.org/10.1137/0913035

[26] Y. Saad, "Iterative Methods for Sparse Linear Systems," 2nd Edition, SIAM, Philadephia, PA, 2003. http://dx.doi.org/10.1137/1.9780898718003

[27] W. Zhang, "Imaging Methods and Computations Based on the Wave Equation,” Science Press, Beijing, 2009. 